\title{
Wormholes and ringholes in a dark-energy universe
}

\author{
Pedro F. González-Díaz \\ Centro de Física "Miguel A. Catalán,” Instituto de Matemáticas y Física Fundamental, Consejo Superior de Investigaciones Científicas, \\ Serrano 121, 28006 Madrid, Spain
}

(Received 18 June 2003; published 31 October 2003)

\begin{abstract}
The effects that the present accelerating expansion of the Universe has on the size and shape of Lorentzian wormholes and ringholes are considered. It is shown that, quite similarly to how it occurs for inflating wormholes, relative to the initial embedding-space coordinate system, whereas the shape of the considered holes is always preserved with time, their size is driven by the expansion to increase by a factor which is proportional to the scale factor of the Universe. In the case that dark energy is phantom energy, which is not excluded by present constraints on the dark-energy equation of state, that size increase with time becomes quite more remarkable, and a rather speculative scenario is here presented where the big rip can be circumvented by future advanced civilizations by utilizing sufficiently grown up wormholes and ringholes as time machines that shortcut the big-rip singularity.
\end{abstract}

DOI: 10.1103/PhysRevD.68.084016

PACS number(s): 04.20.Gz, 98.80.Es

\section{INTRODUCTION}

Solutions to Einstein equations that describe closed timelike curves (CTCs) and time machines have been known since a few years after the discovery of general relativity [1]. At present, apart from a number of interesting recent proposals that have been also much debated [2], essentially three general kinds of such solutions can be recognized. First of all, we have the so-called wormholes [3] and their topological generalizations, ringholes [4] and Klein bottle holes [5]. These correspond to shortcuts of the structure of spacetime itself which are based on topologically generalizing the Misner solution [6] for different matter content that shows what is known as exoticity, i.e., the violation of some classical energy conditions-particularly the dominant energy condition $[3,4,7]$. By far, these are the most popular and studied solutions. Three different topologies have been so far considered for the holes: spherical [3], toroidal [4], and that corresponding to a Klein bottle [5]. The second general kind of spacetime containing CTCs was first introduced by Gott [8] and corresponds to solutions to gravity with infinitely large parallel cosmic strings which move with high velocity relative to each other. These solutions do not entail any kind of exotic matter, but lack physical reality because they cannot be fitted in a finite universe. Finally, Gott and Li have considered [9] the case for the creation of a universe from itself in a sea - presumably the spacetime foam - of nontrivial topological submicroscopic structures where there are CTCs. In particular, a multiply connected de Sitter universe was considered. This was proved to be classically stable and, if the regions containing the CTCs were of Planck size, quantum mechanically stable as well [10]. In this paper we shall restrict ourselves to consider Lorentzian spacetime tunneling which involves just Morris-Thorne wormholes and ringholes.

Semiclassical calculations of the renormalized stressenergy tensor in spacetimes containing CTCs (or, more precisely, satisfying Misner symmetry) generally show a quantum instability at the so-called chronology horizon (i.e., the Cauchy horizon which is the onset of the nonchronal region filled with CTCs), where that tensor diverges [11]. This fact was taken as a basis by Hawking to introduce a much debated generalizing conjecture for chronology protection, stating that the laws of physics prevent CTCs from occurring [12]. It was later argued [13] that while such a conjecture could well apply to macroscopic closed curves, by no means can it be applied to CTCs at the Planck scale-i.e., typically the scale of the quantum spacetime foam. It is now generally recognized that the chronology protection conjecture is just a semiclassical proposal, with no implementation in any spacetime with quantum structure. It would follow that quantum CTCs and time travel are stable quantum mechanically and are pervading the entire spacetime of the vacuum quantum foam. These tentative conclusions will be taken for granted in this paper where we shall investigate the way in which the size of stable wormholes and ringholes, originally at the Planck scale in the spacetime foam, is increased in an accelerating universe, while their initial form is preserved. A similar study was done for inflating wormholes by Roman some years ago [14]. The present research may entail additional interest as (i) it refers to wormholes which are being growth now and (ii) the rate at which the scale factor increases might go beyond the exponential blowup predicted by the presence of a cosmological constant if the equation of state of the Universe is characterized by a parameter $\omega<-1$, a case which still is by no means excluded by the latest observational constraints.

The proper context for the current placement of wormholes and ringholes is that of an accelerating universe, nearly the $70 \%$ of whose energy is in the form of what is known as dark energy - the stuff making up the antigravitational vacuum of the Universe [15]. The latest observations and theoretical developments [16] seem to favor the idea that dark energy might be in the form of either a positive cosmological constant- the first and perhaps yet the favorite explanation for current antigravity dominance-or a slowly varying quintessence scalar field or generalized Chaplygin gas [17], equipped with a perfect-fluid equation of state, $p$ $=\omega \rho$, where $-0.8 \geqslant \omega \geqslant-1.5$. Actually the most reliable constraint on $\omega$ has been provided by the Wilkinson Microwave Anisotropy Probe (WMAP) and is given by $\omega<$ 
$-0.78[18]$. Note that a cosmological constant corresponds to the case $\omega=-1$. Apart from the rather special case of a finite and tiny time-independent cosmological constant [19], up to now there have been two observationally allowed regimes for dark energy which are essentially different. If $\omega$ $<-1$, the energy density of the dark stuff will keep decreasing with time and can induce an ever-accelerating universe of the kind we are able to observe. It has been pointed out that this will inexorably induce the emergence of a future event horizon, so preventing the formulation of any consistent fundamental theory based on defining an $S$ matrix [20]. Even more bizarre are the predictions arising from the regime $\omega<-1$. In this case, there is a violation of the dominant energy condition [21], that is, $p+\rho<0$, which leads to the fact that the energy density increases with time up to an infinite value, at which point the size of the universe blows up at a finite time. This is known as the big rip and represents the end of the universe and everything in it as induced by a kind of dark energy which is denoted as the phantom energy [22]. Of course the problem of a future event horizon in this regime is not only still present but is made even more acute.

Since the dominant energy condition is violated in the regime with $\omega<-1$, the possibility is opened [7] in that regime for the occurrence of the above-discussed wormholes and ringholes. It appears then interesting to investigate the time evolution of such holes when they are embedded in an accelerating universe endowed with dark energy. In particular, we shall study in this paper the way in which the size and shape of wormholes and ringholes evolve with time in such a cosmological scenario. Changes in the hole size are expected to be very much magnified in the case that the dark energy is a phantom energy and will be also considered in the present paper. In addition, we shall explore as well the interesting possibility that Lorentzian holes might be used in the future to circumvent the big rip. The main results of this endeavor are that, in fact, relative to an observer in the initial embedding-space coordinate system, whereas the shape of the holes remains constant along current expansion, their size increases with time at a rate proportional to the cosmic scale factor, and that one might envisage a kind of scenario where sufficiently enlarged holes could be used by future advanced civilizations to escape towards their future from the big rip.

The paper is outlined as follows. In Sec. II we solve the Friedmann equations for an accelerating universe endowed with quintessential dark energy, with and without a positive cosmological constant, where the regime of phantom energy is also considered. The effects of accelerated and "superaccelerated" expansion of the universe on the size and shape of wormholes and ringholes are investigated in detail in Sec. III. Section IV contains a calculation of the stress-energy tensor for both wormholes and ringholes. For the former hole the "exoticity" function [23] is explicitly derived and analyzed. A rather speculative scenario is considered in Sec. V in which wormholes and ringholes are used by future advanced civilizations in order to circumvent the singularity at the big rip. We finally summarize and conclude in Sec. VI, where some short comments are added.

\section{COSMOLOGICAL SOLUTIONS FOR A DARK-ENERGY VACUUM}

In this section I shall derive solutions to the Friedmann equations that correspond to a quintessence scalar field $\phi$, assuming an equation of state with perfect-fluid form,

$$
p=\omega \rho,
$$

where, consistent with present constraints [16], the parameter $\omega$ is assumed to be constant and to take on values within the interval $-2 / 3>\omega \geqslant-3 / 2$, and the pressure $p$ and the energy density $\rho$ are defined by [17]

$$
\begin{aligned}
& p=\frac{1}{2} \dot{\phi}^{2}-V(\phi), \\
& \rho=\frac{1}{2} \dot{\phi}^{2}+V(\phi),
\end{aligned}
$$

with $V(\phi)$ the potential energy for the quintessence field $\phi$ whose precise form is not required in what follows. We see that for a sufficiently slowly varying field $\phi$, we have that $\dot{\phi}^{2}<V(\phi)$, so that the equation-of-state parameter can take on the values specified by the above $\omega$ interval. Inserting then the relation $p=\omega \rho$ in the general expression for cosmic energy conservation, $d \rho=-3(p+\rho) d a / a$ (in which $a$ is the scale factor), and integrating, we obtain, for the energy density,

$$
\rho=R a^{-3(1+\omega)},
$$

where $R$ is an integration constant which should be real and positive. We shall consider in what follows two cases. We regard the cosmic vacuum dark energy either to solely contain the quintessence field $\phi$ or be made by the combination of the quintessence field $\phi$ plus a pure positive cosmological constant $\Lambda$. In the first case, the Friedmann equation reads

$$
\left(\frac{\dot{a}}{a}\right)^{2}=A a^{-3(1+\omega)},
$$

with $A=8 \pi G R / 3$. This differential equation can be easily integrated to yield

$$
a(t)=\left[a_{0}^{3(1+\omega) / 2}+\frac{3(1+\omega) \sqrt{A}}{2}\left(t-t_{0}\right)\right]^{2 /[3(1+\omega)]},
$$

where $a_{0}$ and $t_{0}$ are the initial radius and time, respectively. Note that for $\omega>-1$ this solution describes an accelerating universe whose scale factor increases towards infinity as $t$ $\rightarrow \infty$. The case for $\omega<-1$ is even more interesting. It corresponds to the so-called phantom dark energy for which the dominant energy condition is violated: i.e. [22],

$$
p+\rho<0,
$$

even though the energy density is surprisingly ever increasing. Notice furthermore that in this case the scale factor blows up at a finite time, 


$$
t_{*}=t_{0}+\frac{2}{3(|\omega|-1) a_{0}^{3(|\omega|-1) / 2}},
$$

giving rise to what is now known as a "big rip" [22].

In the most general case where we add to the cosmic quintessence vacuum a positive cosmological constant $\Lambda$, the Friedmann equation would be

$$
\left(\frac{\dot{a}}{a}\right)^{2}=\lambda+A a^{-3(1+\omega)}
$$

where $\lambda=\Lambda / 3$. This equation admits also an analytical solution in closed form [24],

$$
\begin{aligned}
a(t)= & \left(\frac{A}{4 C \lambda}\right)^{1 /[3(1+\omega)]}\left(e^{\frac{3(1+\omega) \sqrt{\lambda}\left(t-t_{0}\right)}{2}}\right. \\
& -C e^{\left.\frac{-3(1+\omega) \sqrt{\lambda}\left(t-t_{0}\right)}{2}\right) 2 /[3(1+\omega)]},
\end{aligned}
$$

where

$$
C=\frac{\sqrt{\lambda+A a_{0}^{-3(1+\omega)}}-\sqrt{\lambda}}{\sqrt{\lambda+A a_{0}^{-3(1+\omega)}}+\sqrt{\lambda}}
$$

with $a_{0}$ the initial scale factor. Since $0<C<1$, we have also in this case the possibility of a big rip at time

$$
t_{*}=t_{0}-\frac{\ln C}{3(|\omega|-1) \sqrt{\lambda}}
$$

in the regime where $\omega<-1$. One would expect that in the vicinity of time $t_{*}$ the size of wormholes and ringholes increased in a rather dramatic way in the two considered cases. We see that in both such cases the larger $a_{0}$ and $|\omega|$, the nearer the doomsday, and hence the time expected to have macroscopic wormholes and ringholes in our universe.

\section{GROWTH OF HOLES IN AN ACCELERATING UNIVERSE}

In this section we shall investigate the way in which the size and form of Lorentzian tunnelings, which can be given as topological four-dimensional generalizations of the Misner space [6] embedded in the current Universe, can be affected by the present accelerating expansion and, in particular, the possibility that there will be a big rip. We shall restrict ourselves to consider two cases. On the one hand, the analysis done by Roman [14] for inflating Morris-Thorne wormholes [3] will here be adapted to study the case of accelerating wormholes. On the other hand, we will also investigate the subtleties that would arise when considering accelerating ringholes, where the tunneling mouths have the topology of a torus [4].

\section{A. Morris-Thorne wormhole}

The Lorentzian metric of the Morris-Thorne wormhole can be given as [3]

$$
d s^{2}=-e^{2 \Phi(r)} d t^{2}+\frac{d r^{2}}{1-\frac{K(r)}{r}}+r^{2} d \Omega_{2}^{2},
$$

where $\Phi$ may be taken to be either zero or a given function of the radial coordinate $r$; otherwise, the function $K(r)$ can be taken either as $K(r)=K_{0}^{2} / r$ for wormholes with zero tidal force or as $K(r)=K_{0}\left[1-\left(1-K_{0}\right) / R_{0}\right]^{2}$ if the exotic matter is confined into an arbitrarily small region around the wormhole throat, and $d \Omega_{2}^{2}=d \theta^{2}+\sin ^{2} \theta d \phi^{2}$ is the metric on the unit two-sphere.

Following Roman [14], we shall now generalize the static metric (3.1) to a time-dependent background metric that describes the time evolution of an initially static wormhole with metric (3.1) immersed in an expanding universe filled with a quintessence field with or without a cosmological constant. This will be done by simply inserting a dimensionless factor proportional to the square of the scale factor into the three-dimensional spatial part of metric (3.1). Taking, for that factor,

$$
g(t)^{2}=\left(1+\frac{3(1+\omega) \sqrt{A}\left(t-t_{0}\right)}{2 a_{0}^{3(1+\omega) / 2}}\right)^{2 /[3(1+\omega)]}
$$

for the pure quintessential case and

$$
g(t)^{2}=\left(e^{3(1+\omega) \sqrt{\lambda}\left(t-t_{0}\right) / 2}-C e^{-3(1+\omega) \sqrt{\lambda}\left(t-t_{0}\right) / 2}\right)^{2 /[3(1+\omega)]}
$$

for the case in which we add a positive cosmological constant to the quintessence field, we have, for the timedependent metric of a wormhole in an accelerating universe,

$$
d s^{2}=-e^{2 \Phi(r)} d t^{2}+g(t)^{2}\left(\frac{d r^{2}}{1-\frac{K(r)}{r}}+r^{2} d \Omega_{2}^{2}\right)
$$

Here the coordinates on the two-sphere have the same geometrical interpretation as for the Morris-Thorne case, and circles of constant $r$ are centered on the throat of the wormhole. Following again Roman [14], the coordinate system is chosen to be comoving with the wormhole geometry, with the throat always located at $r=K_{0}$. Of course, in the limit where $K(r)=\Phi(r)=0$, metric (3.4) reduces to the Friedmann-Robertson-Walker (FRW) metric for an accelerating universe driven by a quintessence field with or without a cosmological constant (see Sec. II), and for $A=0$ and $\lambda$ $=0$ it reduces to the wormhole metric (3.1). If we just set $A=0$, then we would recover the case of an inflating wormhole considered by Roman. We note furthermore that the spacetime described by metric (3.4) is inhomogeneous because of the presence of the wormhole.

Let us next consider how an originally nearly Plancksized wormhole is being enlarged by the accelerating expansion of the universe. The initial wormhole would be a reasonable wormhole at the onset of dark-energy dominance at $t=t_{0}$ for a suitable choice of functions $K(r)$ and $\Phi(r)$, 
which we shall assume. Considering then the proper circumference $c$ at the wormhole throat, $r=K=K_{0}$, for $\theta=\pi / 2$ and any constant time, we have, in the accelerating framework,

$$
c=K_{0} \int_{0}^{2 \pi} d \phi g(t)=2 \pi K_{0} g(t),
$$

which simply is $g(t)$ times the initial circumference, with $g(t)$ given by Eq. (3.2) or Eq. (3.3), depending on whether we do not add or we do add a cosmological constant to the quintessence field. A similar conclusion is also obtained for the radial proper length through the wormhole between any two points $A$ and $B$ at $t=$ const, as that quantity can be computed to be

$$
d(t)= \pm g(t) \int_{r_{A}}^{r_{B}} \frac{d r}{\sqrt{1-K(r) / r}},
$$

which for the simplest wormhole where $K(r)=K_{0}^{2} / r$ becomes

$$
d(t)= \pm g(t)\left(\sqrt{r_{B}^{2}-K_{0}^{2}}-\sqrt{r_{B}^{2}-K_{0}^{2}}\right) .
$$

Thus, both the size of the throat and the radial proper distance between the wormhole mouths increase with $t$ at exactly the same accelerating rate as that of the universe expansion.

That the form of the wormhole metric is preserved with cosmological time can also be easily seen by, e.g., using the embedding [14] of a $t=$ const, $\theta=\pi / 2$ slice of the spacetime (3.4) in a flat three-dimensional Euclidean space with cylindrical metric

$$
d s^{2}=d \bar{z}^{2}+d \bar{r}^{2}+\bar{r}^{2} d \phi^{2} .
$$

Since the metric on the chosen slice is

$$
d s^{2}=g(t)^{2}\left(\frac{d r^{2}}{1-K(r) / r}+r^{2} d \phi^{2}\right),
$$

we get

$$
\begin{gathered}
\bar{r}=\left.g(t) r\right|_{t=\text { const }}, \\
d \bar{r}^{2}=\left.g(t)^{2} d r^{2}\right|_{t=\text { const }} .
\end{gathered}
$$

It follows that, relative to the $\bar{z}, \bar{r}, \phi$ coordinates, the form of the wormhole metric will be preserved provided that the metric on the embedded slice has the form

$$
d s^{2}=\frac{d \bar{r}^{2}}{1-\bar{K}(\bar{r}) / \bar{r}}+\bar{r}^{2} d \phi^{2},
$$

in which $\bar{K}(\bar{r})$ must have a minimum at some $\bar{K}_{0}=\bar{r}_{0}$, such as occurred in the inflating wormhole case [14]. By using Eqs. (3.9) and (3.10) it is easy to see that Eqs. (3.8) and (3.11) can be rewritten into each other if we take

$$
\bar{K}(\bar{r})=g(t) K(r),
$$

which is satisfied by any of the choices for $K(r)$ made explicit by Morris and Thorne [3]. It follows that also a wormhole evolving in a universe filled with dark energy will have the same overall size and shape relative to the $\bar{z}, \bar{r}, \phi$ coordinate system as the initial wormhole had relative to the initial $z, r, \phi$ embedding-space coordinate system. This can be seen if we consider the embedding procedure used by Morris and Thorne-i.e., if we take, as the metric of the embedding space,

$$
d s^{2}=d z^{2}+d r^{2}+r^{2} d \phi^{2} .
$$

Now, since the embedded surface is axially symmetric, $z$ $=z(r)$ and the metric on the embedded surface can be expressed as

$$
d s^{2}=\left[1+\left(\frac{d z}{d r}\right)^{2}\right] d r^{2}+r^{2} d \phi^{2} .
$$

Metrics (3.13) and (3.14) can be identified if (i) the coordinates $r, \phi$ of the embedding space and of the wormhole are identified and (ii) we require

$$
\frac{d z}{d r}= \pm\left(\frac{r}{K(r)}-1\right)^{-1 / 2} .
$$

Applying this procedure to our present case, we have

$$
\frac{d \bar{z}}{d \bar{r}}= \pm\left(\frac{\bar{r}}{\bar{K}(\bar{r})}-1\right)^{-1 / 2}=\frac{d z}{d r}
$$

and, hence,

$$
\begin{aligned}
& \bar{z}(\bar{r})= \pm \int \frac{d \bar{r}}{\sqrt{\frac{\bar{r}}{\bar{K}(\bar{r})}-1}} \pm g(t) \int \frac{d r}{\sqrt{\frac{r}{K(r)}-1}} \\
& =g(t) z(r) \text {, }
\end{aligned}
$$

again as in the Roman's inflating wormhole [14]. Using finally Eqs. (3.10) and (3.17) we then also obtain the same relation between our embedding space at any time $t$ and the initial embedding space at $t=0$; i.e.,

$$
d s^{2}=d \bar{z}^{2}+d \bar{r}^{2}+\bar{r}^{2} d \phi^{2}=g(t)^{2}\left(d z^{2}+d r^{2}+r^{2} d \phi^{2}\right) .
$$

Using Eqs. (3.9), (3.10), (3.12), and (3.16) we also obtain, for the Morris-Thorne flareout condition [3],

$$
\begin{gathered}
\frac{d^{2} \bar{r}(\bar{z})}{d \bar{z}^{2}}=g(t)^{-1}\left(\frac{K-K^{\prime} r}{2 K^{2}}\right), \\
g(t)^{-1}\left(\frac{d^{2} r(z)}{d z^{2}}\right)=\frac{\bar{K}-\bar{K}^{\prime} \bar{r}}{2 \bar{K}^{2}}>0,
\end{gathered}
$$

at or near the throat. In obtaining this condition use has been made of $\bar{K}(\bar{r})^{\prime}=d \bar{K} / d \bar{r}=K(r)^{\prime}=d K / d r$. 


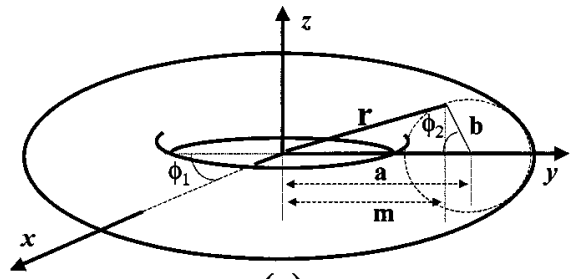

(a)

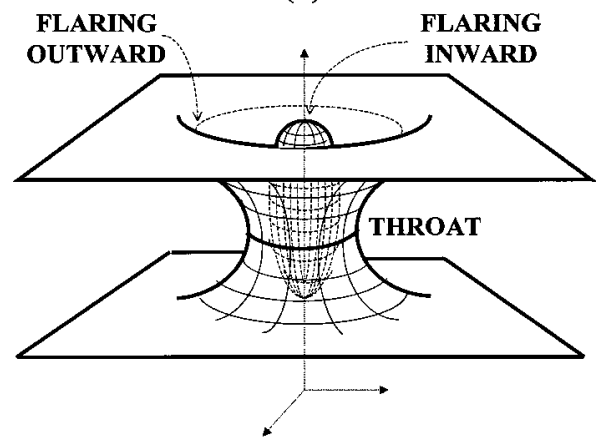

(b)

FIG. 1. (a) Coordinates and geometric parameters on the twotorus; any point on the torus surface can be labeled by parameters $a$, $b, \phi_{1}$, and $\phi_{2}$. (b) Embedding diagram for the spacetime of a ringhole connecting two asymptotically flat regions.

The conclusion is thus obtained that, whereas the shape of a wormhole naturally existing in an accelerating universe is preserved with time, its size is increased relative to the initial embedding-space coordinate system. We note that if the equation of state for dark energy is characterized by a parameter $\omega>-1$, then the size increase will be slower than in an inflating universe, but if the wormhole is placed in a universe filled with phantom energy for which $\omega<-1$ (which is the most natural situation for a wormhole to exist because in this case the required energy exoticity for the wormhole existence is shared by the overall universe), then the wormhole will increase size more rapidly than in the inflating case, which is characterized by the parameter $\omega=-1$, which corresponds to a cosmological constant.

\section{B. Ringhole}

The case of a ringhole entails certain subtleties that make the treatment of its time dependence in an accelerating universe somehow different from the case of an inflating wormhole. A ringhole is nothing but a wormhole with the spherical topology being replaced for a toroidal topology; that is, it is just a four-dimensional Misner space where the planes are substituted by tori whose relevant geometric parameters are given in Fig. 1(a). The embedding of a ringhole in flat regions of the universe is depicted in Fig. 1(b), where we point out the throat position and how the inner and outer surfaces flare, respectively, inward and outward near the throat. The general Lorentzian metric for a ringhole can be written as

$$
\begin{aligned}
d s^{2} & =-e^{2 \Phi(r)} d t^{2}+\frac{d r^{2}}{1-\frac{K(b)}{b}}+m^{2} d \phi_{1}^{2}+b^{2} d \phi_{2}^{2} \\
& =-e^{2 \Phi(r)} d t^{2}+\frac{n^{2}}{r^{2}} d \ell^{2}+m^{2} d \phi_{1}^{2}+b^{2} d \phi_{2}^{2},
\end{aligned}
$$

where $\phi_{1}$ and $\phi_{2}$ are the angles which usually define the position on the torus [see Fig. 1(a)],

$$
\begin{aligned}
& b=\sqrt{b_{0}^{2}+\ell^{2}}, \\
& m=a-b \cos \phi_{2}, \\
& n=b-a \cos \phi_{2}, \\
& r=\sqrt{a^{2}+b^{2}-2 a b \cos \phi_{2}},
\end{aligned}
$$

$a$ is the constant radius of the axial circumference defined by the torus, and $b$ is the radius of the circular transversal section on the torus, with $b_{0}$ giving that radius at the throat.

Following the same procedure as for the Morris-Thorne wormhole, we can now write the ringhole metric generalized to a time-dependent accelerating dark-energy background in the form

$$
d s^{2}=-e^{2 \Phi(r)} d t^{2}+g(t)^{2}\left(\frac{d r^{2}}{1-\frac{K(b)}{b}}+m^{2} d \phi_{1}^{2}+b^{2} d \phi_{2}^{2}\right),
$$

with $g(t)$ as given by either Eq. (3.3) or Eq. (3.2), depending on whether we introduce or not a cosmological constant among the components of the dark energy. Because of the presence of the ringhole, the spacetime described by metric (3.25) is inhomogeneous as well. Also in this case the metric reduces to the FRW metric for an accelerating universe in the limit $K(b)=\Phi(r)=0$ and becomes the ringhole metric (3.20) for $A=0, \lambda=0$ simultaneously. If we would just set $A=0$, then we have an inflating ringhole evolving in a de Sitter-like space.

In what follows we apply the analysis done for wormholes to the case of ringholes. In the latter case we have two proper circumferences $c_{\min }$ and $c_{\max }$ at the throat. They are defined by $c_{\min }: \ell=0, \phi_{2}=0$ and $c_{\max }: \ell=0, \phi_{2}=\pi$, both at any time $t=$ const. We then have

$$
\begin{gathered}
c_{\min }=\int_{0}^{2 \pi} g(t)\left(a-b_{0}\right) d \phi_{1}=2 \pi g(t) r_{\min }=2 \pi g(t) m_{\min }, \\
c_{\max }=\int_{0}^{2 \pi} g(t)\left(a+b_{0}\right) d \phi_{1}=2 \pi g(t) r_{\max }=2 \pi g(t) m_{\max },
\end{gathered}
$$

where the subscript "min" denotes $\phi_{2}=0$ and the subscript "max" denotes $\phi_{2}=\pi$, in the corresponding expressions for $m$ and $r$. Thus, one obtains that both circumferences are equally increased by a factor $g(t)$.

The radial proper length through a ringhole between any two points $A$ and $B$ at any constant time and constant $\phi_{2}$ is similarly increased by a factor $g(t)$ generally according to 


$$
d(t)= \pm g(t) \int_{r_{A}}^{r_{B}} \frac{d r}{\sqrt{1-\frac{K(b)}{b}}},
$$

which once again is just $g(t)$ times the initial radial proper separation. In case of separation along the ringhole inner surface $\phi_{2}=0$, if we assume the simplest ringhole choice $K=K_{0}^{2} / b$ such that $K_{0} \ll(a-r)$, we obtain the approximate expression

$$
d(t) \simeq g(t)\left(r_{B}-r_{A}\right)\left[1+\frac{K_{0}^{2}}{2\left(a-r_{B}\right)\left(a-r_{A}\right)}\right] .
$$

For the same ringhole choice but now with $K_{0} \ll(r-a)$, we would have, for separation distances along the outer surface $\phi=\pi$,

$$
d(t) \simeq g(t)\left(r_{B}-r_{A}\right)\left[1+\frac{K_{0}^{2}}{2\left(r_{B}-a\right)\left(r_{A}-a\right)}\right] .
$$

The conclusion is then that both the distinct geometrical sizes defined on the ringhole throat and the radial proper distances defined along any ringhole surface between the two mouths increase with time following the same pattern.

In order to investigate whether the form of a ringhole is also preserved with time in the accelerating background, let us again embed in a flat three-dimensional Euclidean space with metric (3.13) either a slice $t=$ const, $\phi_{2}=0$, or a slice $t=$ const, $\phi_{2}=\pi$. On the first of such slices one would have

$$
d s^{2}=g(t)^{2}\left(\frac{d r^{2}}{1-\frac{K(b)}{b}}+m^{2} d \phi_{1}^{2}\right) .
$$

Comparing Eq. (3.13) with Eq. (3.31), we have

$$
\begin{gathered}
\bar{r}=\bar{m}=\left.g(t) r\right|_{t=\mathrm{const}}=\left.g(t) m\right|_{t=\mathrm{const}}, \\
d \bar{r}=d \bar{m}=\left.g(t) d r\right|_{t=\mathrm{const}}=\left.g(t) d m\right|_{t=\mathrm{const}},
\end{gathered}
$$

with

$$
r=r_{\text {min }}=m=m_{\min }=a-b .
$$

On the other extreme slice $\phi_{2}=\pi$ we obtain the same expressions (3.32) and (3.33), but now referred to the maximal values of parameters $r$ and $m$ : i.e.,

$$
r=r_{\max }=m=m_{\max }=a+b .
$$

It follows that expressions (3.32) and (3.33) are valid for any slice $t=$ const, from $\phi_{2}=0$ to $\phi_{2}=\pi$. Hence,

$$
\bar{n}=g(t) n, \quad \bar{a}=g(t) a, \quad \bar{b}=g(t) b,
$$

for any of such slices.

Thus, with respect to the $\bar{z}, \bar{r}, \phi$ coordinates, the form of the ringhole metric will be preserved if the metric on the embedded slice is written as

$$
d s^{2}=\frac{d \bar{r}^{2}}{1-\frac{\bar{K}(\bar{b})}{\bar{b}}}+\bar{m}^{2} d \phi_{1}^{2},
$$

where $\bar{K}(\bar{b})$ is assumed to have a minimum at some $\bar{K}\left(\bar{b}_{0}\right)$ $=\bar{K}_{0}$. We can rewrite Eq. (3.35) in the form

$$
d s^{2}=g(t)^{2}\left(\frac{d r^{2}}{1-\frac{K(b)}{b}}+m^{2} d \phi_{1}^{2}\right)
$$

by using Eqs. (3.32)-(3.34) and

$$
\bar{K}(\bar{b})=g(t) K(b) .
$$

Choosing for the ringhole one which is defined to contain exotic material everywhere, that is,

$$
K(b)=K_{0}^{2} / b,
$$

and rewriting the right-hand side of expression (3.38) in terms of $\bar{b}$ as given in Eq. (3.34), by using Eq. (3.32) we can see that expression (3.37) is satisfied provided the third equation of Eqs. (3.34) is satisfied and vice versa.

It follows that the enlarged ringhole will also have the same overall size and shape relative to coordinates $\bar{z}, \bar{r}, \phi$, as the initial ringhole had relative to the initial $z, r, \phi$ embedding-space coordinates. This can be most clearly seen by pointing out that our embedding scheme corresponds to one whose $z, r$ coordinates scale with time, such as can be shown following the embedding procedure given in Ref. [4] for Eqs. (3.7) and (3.35). Using the above expressions for relating the ringhole parameters, one can get

$$
\frac{d \bar{z}}{d \bar{r}}= \pm\left(\frac{\bar{b}}{\bar{K}(\bar{b})}-1\right)^{-1 / 2}=\frac{d z}{d r}
$$

which implies

$$
\begin{aligned}
\bar{z}(\bar{r}) & = \pm \int \frac{d \bar{r}}{\sqrt{\frac{\bar{b}}{\bar{K}(\bar{b})}-1}}= \pm g(t) \int \frac{d r}{\sqrt{\frac{b}{K(b)}-1}} \\
& =g(t) z(r) .
\end{aligned}
$$

Note that if we take for the ringhole a homogeneous distribution of exotic matter, then, on the surface $\phi_{2}=0$,

$$
z(r)=\mp K_{0} \ln \left(b+\sqrt{b^{2}-K_{0}^{2}}\right),
$$

and the same expression with reversed sign on the surface $\phi_{2}=\pi$. Therefore the relation between our embedding space, at any time $t$ and any value of the angular coordinate $\phi_{2}$, and the initial embedding space at $t=0$ and the same chosen value of $\phi_{2}$ has the same form as that is given by Eq. (3.18) for wormholes. Thus, relative to the barred coordinates, the ringhole does not change size because the scaling 
of the embedding space exactly compensates the expansion of the ringhole, such as it also occurred for wormholes.

The analogous expressions of the flareout and flarein conditions of the initial ringhole [4] are, respectively, given at any nonzero time $t$ by

$$
\begin{aligned}
& \frac{d^{2} \bar{r}}{d \bar{z}^{2}}>0, \quad 2 \pi-\phi_{2}^{c}>\phi_{2}<\phi_{2}^{c}, \\
& \frac{d^{2} \bar{r}}{d \bar{z}^{2}}<0, \quad-\phi_{2}^{c}>\phi_{2}<\phi_{2}^{c},
\end{aligned}
$$

at or near the throat, with $\phi_{2}^{c}=\arccos (b / a)$ at the angular horizons [4]. From the set of the above equations, one has

$$
\begin{aligned}
\frac{d^{2} \bar{r}}{d \bar{z}^{2}} & =\frac{\bar{r} \bar{K}(\bar{b})-\bar{K}(\bar{b})^{\prime} \bar{b} \bar{n}}{\bar{n} \bar{K}(\bar{b})^{2}}=\frac{d^{2} r(z)}{g(t) d z^{2}} \\
& =\frac{r K(b)-K(b)^{\prime} b n}{g(t) n k(b)^{2}}>0
\end{aligned}
$$

for the flareout condition and

$$
\begin{aligned}
\frac{d^{2} \bar{r}}{d \bar{z}^{2}} & =\frac{\bar{r} \bar{K}(\bar{b})-\bar{K}(\bar{b})^{\prime} \bar{b} \bar{n}}{\bar{n} \bar{K}(\bar{b})^{2}}=\frac{d^{2} r(z)}{g(t) d z^{2}} \\
& =\frac{r K(b)-K(b)^{\prime} b n}{g(t) n k(b)^{2}}<0
\end{aligned}
$$

for the flarein condition. In these expressions $\bar{K}^{\prime}=d \bar{K} / d \bar{r}$ and $K^{\prime}=d K / d r$. It follows that the flareout and flarein conditions relative to the barred quantities have the same form as those for static ringholes. With respect to the nonbarred quantities, whereas the flareout condition appears as though it might be harder to satisfy as time goes on, the flarein condition looks as though it were easier to satisfy as time goes on. This is, however, due to the fact that as the ringhole increases, both its throat size and any proper length also increase along with the surrounding space. The ringhole then needs to flare out less and less on the outer surfaces near the throat and to flare in more and more on the inner surfaces near the throat, as the two external spaces which are connected by the ringhole move farther apart.

We thus again obtain for ringholes in an accelerating universe the conclusion that, relative to the initial embeddingspace coordinate system, the size of the hole will increase with time without undergoing any deformation whatsoever. If dark energy is governed by an equation of state with parameter $\omega>-1$, then that increase of hole size will be once again slower than for inflating holes, but if $\omega<-1$, the ringhole will "superinflate," with the smaller $\omega$, the larger the expansion rate of the ringhole. Since it was shown [4] that, even near their throat, the internal channel of these ringholes possesses certain itineraries through which a traveler would not find any exotic matter, one could envisage a big-rip scenario for which initially submicroscopic ringholes could be grown to macroscopic sizes through which future inhabitants of the universe might find themselves saved just before the big rip started to smash their worlds. A more detailed proposal about this possibility will be considered in Sec. V.

\section{STRESS-ENERGY TENSOR AND EXOTICITY}

Using the notation that stems from defining the set of orthonormal basis vectors,

$$
\begin{aligned}
& e_{\hat{t}}=e^{-\Phi} e_{t}, \quad e_{\hat{r}}=\frac{\sqrt{1-K(r) / r}}{g(t)} e_{r}, \\
& e_{\hat{\theta}}=\frac{e_{\theta}}{r g(t)}, \quad e_{\hat{\phi}}=\frac{e_{\phi}}{g(t) r \sin \theta},
\end{aligned}
$$

for wormholes, and

$$
e_{\hat{t}}=e^{-\Phi} e_{t}, \quad e_{r}=\frac{\sqrt{1-K(b) / b}}{g(t)} e_{r}
$$

$$
e_{(\hat{\phi})_{1}}=\frac{\left(e_{\phi}\right)_{1}}{m g(t)}, \quad e_{(\hat{\phi})_{2}}=\frac{\left(e_{\phi}\right)_{2}}{b g(t)}
$$

for ringholes, from the Einstein equation

$$
R_{\hat{\mu} \hat{\nu}}-\frac{1}{2} g_{\hat{\mu} \hat{\nu}} R=8 \pi T_{\hat{\mu} \hat{\nu}},
$$

we obtain the components of the stress-energy tensor (incorporating in them the dark-energy vacuum contributions). In the most general case that we take for $g(t)$, expression (3.3) for an expanding wormhole, these components are

$$
\begin{aligned}
T_{\hat{t} \hat{t}}= & \frac{1}{8 \pi[-]^{2}}\left(\frac{4}{3} C_{1}^{2}[+]^{2}+\frac{K^{\prime}}{r^{2}}\right)=\rho(r, t), \\
T_{\hat{r} \hat{r}}= & \frac{1}{8 \pi}\left\{-\frac{2}{3} C_{1}^{2}\left(\frac{\frac{5}{3}[+]^{2}}{[-]^{2}}+(1+\omega)\right)\right. \\
& -\frac{\left.\frac{K}{r^{2}}-2 \Phi^{\prime}\left(1-\frac{K}{r}\right)\right)}{\left.r[-]^{4 / 3(1+\omega)}\right\}} \\
= & -\tau(r, t), \\
T_{\hat{t} \hat{r}}= & \frac{4 C_{1}[+] e^{-\Phi} \Phi^{\prime} \sqrt{1-\frac{K}{r}}}{24 \pi[-]^{(5+3 \omega) / 3(1+\omega)}} \\
= & -f(r, t),
\end{aligned}
$$




$$
\begin{aligned}
8 \pi T_{\hat{\theta} \hat{\theta}}= & 8 \pi T_{\hat{\phi} \hat{\phi}}=8 \pi p(r, t) \\
= & \left\{\frac{4}{3} C_{1}^{2}\left(\frac{\omega[+]^{2}}{[-]^{2}}-(1+\omega)\right) e^{-2 \Phi}\right. \\
& -[-]^{-4 / 3(1+\omega)}\left[\frac{1}{2}\left(\frac{K}{r^{3}}-\frac{K^{\prime}}{r^{2}}\right)\right. \\
& \left.\left.+\frac{\Phi^{\prime}}{r}\left(1-\frac{K}{2 r}-\frac{K^{\prime}}{2}\right)+\left(1-\frac{K}{r}\right)\left[\Phi^{\prime \prime}+\left(\Phi^{\prime}\right)^{2}\right]\right]\right\} \\
= & p(r, t),
\end{aligned}
$$

where

$$
[ \pm]=e^{C_{1}(1+\omega) t} \pm C_{2} e^{C_{1}(1+\omega) t} .
$$

In the case of a ringhole and $g(t)$ given by Eq. (3.3), we get, instead,

$$
\begin{aligned}
& T_{\hat{t} \hat{t}}=T_{\hat{t} \hat{t}}(t=0)+\frac{C_{1}^{2}[+]^{2}}{12 \pi[-]^{2}}=\rho(r, t), \\
& T_{\hat{r} \hat{r}}=T_{\hat{r} \hat{r}}(t=0)-\frac{C_{1}^{2}}{12 \pi}\left(\frac{\frac{5}{3}[+]^{2}}{[-]^{2}}+(1+\omega)\right)=-\tau(r, t), \\
& T_{\hat{t} \hat{r}}=T_{\hat{t} \hat{r}}(t=0)+\frac{C_{1} e^{-\Phi} \Phi^{\prime} \sqrt{1-\frac{K}{b}}[+]}{6 \pi[-]^{(5+3 \omega) / 3(1+\omega)}}=-f(r, t),
\end{aligned}
$$

$$
\begin{aligned}
T_{\hat{\phi}_{1} \hat{\phi}_{1}} & =T_{\hat{\phi}_{2} \hat{\phi}_{2}}=T_{\hat{\phi}_{1} \hat{\phi}_{1}}(t=0)+\frac{C_{1}^{2} e^{-2 \Phi}}{6 \pi}\left(\frac{\omega[+]^{2}}{[-]^{2}}-(1+\omega)\right) \\
& =p(r, t),
\end{aligned}
$$

where $[+]$ and $[-]$ are time-dependent functions which are again defined in Eq. (4.9). In these equations $\rho, \tau, f$, and $p$ are the mass-energy density, the radial tension per unit area, the energy flux in the radial direction, and the lateral pressures, as measured by observers stationed at constant values of the coordinates $\left(r, \theta, \phi\right.$ or $\left.r, \phi_{1}, \phi_{2}\right)$, respectively. The terms denoted by $T_{\hat{\mu}} \hat{\nu}(t=0)$ for the ringhole case correspond to those of the static case and can easily be derived from those provided in Ref. [4]. The energy fluxes in the radial direction given by Eqs. (4.7) and (4.12) for wormholes and ringholes, respectively, will vanish at the throat in both cases, such as one should expect. More surprising is the feature that all the components of the stress-energy tensor will diverge as one approaches the critical big-rip time, $t \rightarrow t_{*}$, if $\omega<-1$, both for wormholes and ringholes. This is an interesting result that is related with the known fact that the vacuum energy density increases with time, tending to infinity at the big rip, for phantom-energy quintessential models [20]. Assuming, on the other hand, that $\Phi(r) \rightarrow 0$ and either $K / r \rightarrow 0$ or
$K / b \rightarrow 0$, as $r \rightarrow \infty$, the stress-energy components asymptotically recover their pure cosmological dark-energy forms which also contained a cosmological constant. Had we used the expansion factor given by Eq. (3.2) in assuming that limit, then we had obtained stress-energy components in terms of the quintessence-field parameters only. Finally, for the particularly simple example that one chooses holes such that $\Phi(r)=0$ everywhere, we see that the energy flux in the radial direction becomes identically zero for both kinds of holes, as should also be expected.

In what follows of the present section we shall restrict ourselves to briefly discuss the exotic character of the matter entering the holes. For the sake of simplicity, we confine our analysis to the case of wormholes, but note that all the results we obtain for this case are directly applicable to the ringhole case. In order to describe the exotic nature of the matter in these holes, an "exoticity" function has been introduced which is generally defined by [23]

$$
\xi=\frac{\tau-\rho \mp f}{|\rho|} .
$$

In the case of a wormhole the exoticity function can be written in the explicit form

$$
\begin{aligned}
\xi= & \left\{-\left[-\frac{2}{3} C_{1}^{2}\left(\frac{5}{3}[+]^{2}+(1+\omega)[-]^{2}\right)-\Psi_{1}(r)\right]\right. \\
& \left.-\left(\frac{4}{3} C_{1}^{2}[+]^{2}+\frac{K^{\prime}}{r^{2}}\right)\right\}\left|\frac{4}{3} C_{1}^{2}[+]^{2}+\frac{K^{\prime}}{r^{2}}\right|^{-1} \pm \Psi_{2}(r),
\end{aligned}
$$

where

$$
\begin{aligned}
& \Psi_{1}=\left(\frac{K}{r^{2}}-22 \Phi^{\prime}\left(1-\frac{K}{r}\right)[-]^{2(1+3 \omega) / 3(1+\omega)}\right), \\
& \Psi_{2}=\frac{4 e^{-\Phi} \Phi^{\prime} \sqrt{1-\frac{K}{r}} C_{1}[+][-]^{(1+3 \omega) / 3(1+\omega)}}{3\left|\frac{4}{3} C_{1}^{2}[+]^{2}+\frac{K^{\prime}}{r^{2}}\right|} .
\end{aligned}
$$

Equation (4.15) is generally nonzero, and hence the exotic character of the matter in the wormhole is also generally preserved along evolution. In case that dark energy is phantom energy, i.e., if $\omega<-1$, however, as one approaches the big rip at $t=t_{*}=-\ln \left(C_{2}\right) /\left[2 C_{1}(|\omega|-1)\right]$, Eq. (4.15) reduces to the approximate expression

$$
\xi_{\mathrm{ph}} \simeq \frac{\frac{K^{\prime}}{r^{2}}-\frac{16}{9} C_{1}^{2} C_{2}}{\left|\frac{K^{\prime}}{r^{2}}+\frac{16}{3} C_{1}^{2} C_{2}\right|}
$$


We can now see that the matter entering a wormhole approaching the big rip may be of two different kinds, as related to the cosmological parameters of the universe where the hole is embedded in. If the wormhole-defining parameter $K \neq K_{0}+16 C_{1}^{2} C_{2} r^{3}$, where $K_{0}$ is an arbitrary constant, then the matter is still exotic in the neighborhood of the big rip. However, if the wormhole is defined so that $K \simeq K_{0}$ $+16 C_{1}^{2} C_{2} r^{3}$, then there will be a time as we approach the big rip at which all the matter in the wormhole behaved as though it were ordinary matter, without any violation of the energy conditions. As was pointed out before, these results also apply to the case of a ringhole when it approaches the big rip.

\section{ESCAPING TOWARDS THE FUTURE FROM THE BIG RIP?}

The content of this section has a rather speculative character. We shall deal with the big-rip scenario, suggested by Caldwell, Kamionkowsni, and Weinberg [22], for any of the two cosmological scenarios considered in Sec. II, whenever the parameter of the equation of state is $\omega<-1$. Depending on the precise values assumed for $\omega$ and the initial size $a_{0}$, as the doomsday is being approached, one would expect astrophysical objects, such as clusters and galaxies, next the solar system, and then the Earth itself and all its constituents and living inhabitants, to successively disappear beyond the horizon of any observer. At the big rip not even quarks and electrons are left in the observable universe, so that everything would disappear. The doomsday seems thus unavoidable in this case. However, there could have a way out from this catastrophe.

Actually, the solution described by Eqs. (2.6) and (2.10) shows two branches around the critical time $t_{*}$, one along which the expansion of the universe dramatically accelerates towards the singularity at $t_{*}$ and the other for $t>t_{*}$, which describes a universe which exponentially decelerates towards zero size as $t \rightarrow \infty$. There is a possibility to save any animated or inanimated structure assuming the natural or technological embarking, at the "last minute" before the final catastrophe, of all these structures in wormholes and ringholes (grown up by the accelerated expansion to macroscopic sizes from the submicroscopic constructs that originally pervaded the gravitational vacuum) with one mouth opening to the expanding branch and the other opening to the later contracting branch. Note that since one of the hole mouths would be expanding and the other mouth would be contracting, every such hole behaved as though it were a true time machine, so that the animated or inanimated beings embarking these holes before the big rip would travel into their future. The big rip would then be shortcut and the travelers suddenly found themselves evolving in a contracting universe (Fig. 2) where all parts that went beyond their horizon-possibly galaxies, clusters, etc.--as they approached the big rip, should successively reappear as time progressed in the future.

At least three conditions are needed in order for such a travel to be feasible: (1) the holes must be enlarged enough to allow travelers to fit in them, (2) the travelers should not

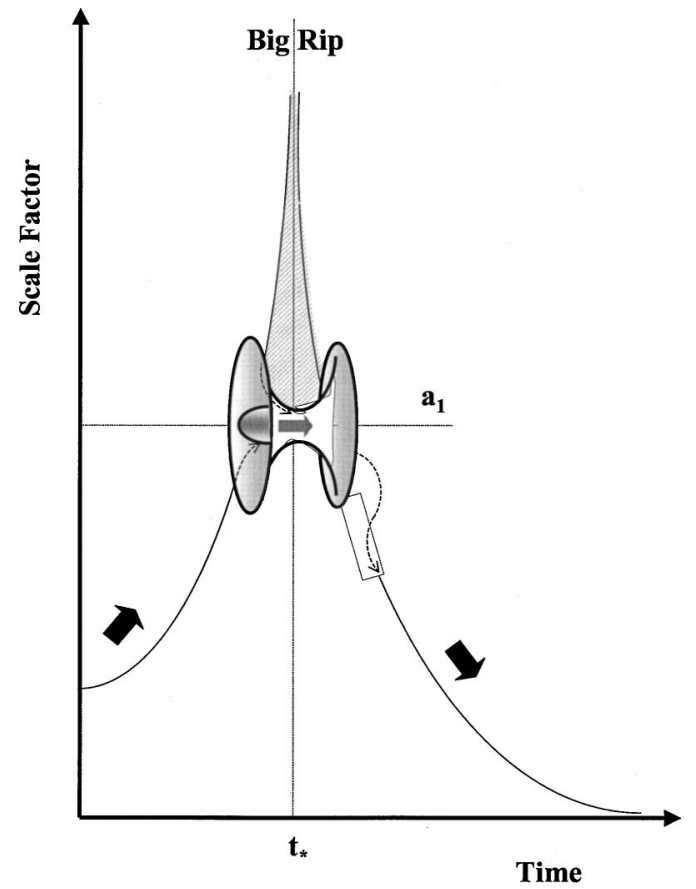

FIG. 2. Pictorial representation of the itinerary followed by a traveler who evolves in an accelerating universe with phantom energy, avoiding the big rip through a shortcut made of a ringhole.

find a remarkable quantity of exotic matter while traversing the hole, and (3) the value of the scale factor at the embarking time on the expanding branch should be the same as the value of that factor at the arrival time on the contracting branch. Condition (1) can be fulfilled if travel takes place at a time sufficiently close to the big rip which, at the same time, would be early enough to keep the travelers and their surrounding structures reasonably intact. At the end of Sec. IV it was seen that, at least for a given type of hole, the exotic character of the hole matter becomes sufficiently weakened so as to allow condition (2) to be satisfied. On the other hand, Visser, Kar, and Dadhich have quite recently shown [25] that there exist spacetime geometries containing traversable wormholes which are supported by arbitrarily small quantities of exotic matter. Finally and quite independent of these features, if we choose ringholes to play the role of the time machine, then there are specific itineraries through the tunneling along which the traveler does not find any exotic matter [4].

For the case of wormholes in a universe filled with quintessential dark energy plus a positive cosmological constant, condition (3) would require the embarking (-) and arrival $(+)$ times to be given by

$$
\begin{aligned}
t_{ \pm}= & \frac{1}{2 C_{1}(|\omega|-1)} \ln \left[\frac{1}{C}+\frac{B}{2 a_{1}^{3(|\omega|-1)} C^{2}}\right. \\
& \left.\times\left(1 \pm \sqrt{1+\frac{4 C a_{1}^{3(|\omega|-1)}}{B}}\right)\right],
\end{aligned}
$$

where $a_{1}$ is any finite sufficiently large value of the scale factor, 


$$
C_{1}=3(|\omega|-1) \sqrt{\lambda} / 2
$$

and

$$
B=A /(4 C \lambda)
$$

We have then

$$
t_{-}<t_{*}<t_{+} \cdot
$$

Figure 2 pictorially shows the itinerary followed by the content of the universe which shortcuts the big rip and a region around it through a ringhole. It has been recently suggested [26] that there could be some models for phantom dark energy for which no big rip takes place. The discussion included in this section opens the possibility that even though such models were shown to be incorrect and phantom energy then necessarily implied a big rip, the universe could still have in store gravitational procedures that allowed its inhabitants and, actually, its whole content to circumvent the big rip, while conquering an additional infinite period of evolution where some laws of physics might be reversed.

\section{SUMMARY AND CONCLUSIONS}

In this paper we have dealt with the two simplest topologically different four-dimensional generalizations of Misner space when embedded in an accelerating universe filled with dark energy satisfying a general perfect-fluid equation of state, $p=\omega \rho$, where $-0.8 \geqslant \omega \geqslant-1.5$, according to the most recent constraints [16]. We investigate how the overall size and precise shape of wormholes and ringholes are in this way induced to evolve due to the rapid expansion of the universe. This study is carried out adding to the usual quintessence model with constant $\omega>-1$ the phantom-energy regime where $\omega<-1$ which is not excluded by present observations. It is obtained that whereas the shape of the holes is always preserved, their size increases with time relative to the initial embedding-space coordinate system. With respect to that system, the exoticity function is also seen to vary with time, though it generally does not vanish anywhere, even when a big-rip model is considered. The above results allow us to tentatively consider a scenario where, even though the big rip is taken to be unavoidable for $\omega<-1$, possible future civilizations might still escape from the doomsday toward their future by using sufficiently grown-up wormholes and ringholes as time machines. In such a scenario the suggested difficulty for constructing fundamental theories based on defining an $S$ matrix could be also circumvented as there will be no future event horizon for the contracting branch of cosmological evolution that follows the big rip.

\section{ACKNOWLEDGMENTS}

The author thanks Mariam Bouhmadi and Carmen L. Sigüenza for useful discussions. This work was supported by DGICYT under Research Project No. BMF2002-03758.
[1] K. Lanczos, Z. Phys. 21, 73 (1924); W.J. van Stockum, Proc. R. Soc. Edinburgh 57, 135 (1937); K. Gödel, Rev. Mod. Phys. 21, 447 (1949); C.W. Misner, in Relativity Theory and Astrophysics I. Relativity and Cosmology, edited by J. Ehlers (American Mathematical Society, Providence, RI, 1967).

[2] M. Cataldo, P. Salgado, and P. Minning, Phys. Rev. D 66, 124008 (2002); N.A. Lemos and G.A. Monerat, Gen. Relativ. Gravit. 35, 423 (2003); S.V. Sushkov and S.-W. Kim, Class. Quantum Grav. 19, 4909 (2002); C. Hellaby and A. Krasinski, Phys. Rev. D 66, 084011 (2002).

[3] M.S. Morris, K.S. Thorne, and M. Yursever, Phys. Rev. Lett. 61, 1446 (1988); M.S. Morris and K.S. Thorne, Am. J. Phys. 56, 395 (1998).

[4] P.F. González-Díaz, Phys. Rev. D 54, 6122 (1996).

[5] P.F. González-Díaz and L.J. Garay, Phys. Rev. D 59, 064026 (1999).

[6] S.W. Hawking and G.F.R. Ellis, The Large-Scale Structure of Space-Time (Cambridge University Press, Cambridge, UK, 1973).

[7] M. Visser, Lorentzian Wormholes (AIP, Woodbury, 1995); K.S. Thorne, Black Holes and Time Warps (Norton, New York, 1994).

[8] J.R. Gott, Phys. Rev. Lett. 66, 1126 (1991).

[9] J.R. Gott and Li-Xin Li, Phys. Rev. D 58, 023501 (1998).

[10] P.F. González-Díaz, Phys. Rev. D 59, 123513 (1999).

[11] W.A. Hiscock and D.A. Konkowski, Phys. Rev. D 26, 1225 (1982).
[12] S.W. Hawking, Phys. Rev. D 46, 603 (1992).

[13] P.F. González-Díaz, Phys. Rev. D 58, 124011 (1998).

[14] T.A. Roman, Phys. Rev. D 47, 1370 (1993).

[15] M.S. Turner, "Dark energy and the new cosmology," contribution to the SNAP Yellow Book, Snowmass, 2001, astro-ph/0108103; S.L. Bridle, O. Lahav, J.P. Ostriker, and P.J. Steinhardt, Science 299, 1532 (2003); T. Padmanabhan, Phys. Rep. 380, 235 (2003); M. Carmeli, "Accelerating universe, Cosmological Constant and Dark Energy," astro-ph/0111259. For reviews and an extensive collection of papers on dark energy see the website www.to.infn giunti/NU/Cosmology/

[16] S. Perlmutter et al., Astrophys. J. 483, 565 (1997); The Supernova Cosmology Project, S. Perlmutter et al., Nature (London) 391, 51 (1998); P.M. Garnavich et al., Astrophys. J. Lett. 493, L53 (1998); B.P. Schmidt, Astrophys. J. 507, 46 (1998); A.G. Riess et al., Astron. J. 116, 1009 (1998); A. Riess et al., Astrophys. J. 560, 49 (2001); A.C. Baccigalupi, A. Balbi, S. Matarrase, F. Perrotta, and N. Vittorio, Phys. Rev. D 65, 063520 (2002); M. Melchiorri, L. Mersini, C.J. Odman, and M. Tradden, ibid. 68, 043509 (2003); M. Doupis, A. Riazuelo, Y. Zolnierowski, and A. Blanchard, Astron. Astrophys. 405, 409 (2003).

[17] C. Wetterich, Nucl. Phys. B302, 668 (1988); J.C. Jackson and M. Dodgson, Mon. Not. R. Astron. Soc. 297, 923 (1998); J.C. Jackson, ibid. 296, 619 (1998); R.R. Caldwell, R. Dave, and P.J. Steinhardt, Phys. Rev. Lett. 80, 1582 (1998); L. Wang and P.J. Steinhardt, Astrophys. J. 508, 483 (1998); R.R. Caldwell 
and P.J. Steinhardt, Phys. Rev. D 57, 6057 (1998); G. Huey, L. Wang, R. Dave, R.R. Caldwell, and P.J. Steinhardt, ibid. 59, 063005 (1999); P.F. González-Díaz, ibid. 62, 023513 (2000); P.J. Steinhardt, L. Wang, and I. Zlatev, Phys. Rev. Lett. 82, 896 (1999); Phys. Rev. D 59, 123504 (1999); I. Zlatev and P.J. Steinhardt, Phys. Lett. B 459, 570 (1999); A. Kamenshchik, U. Moschella, and V. Pasquier, ibid. 511, 265 (2001); N. Bilic, G.B. Tupper, and R. Viollier, ibid. 535, 17 (2001); M.C. Bento, O. Bertolami, and A.A. Sen, Phys. Rev. D 66, 043507 (2002); J.S. Fabris, S.V. Gonzalves, and P.E. de Souza, Gen. Relativ. Gravit. 34, 53 (2002); V. Gorini, A. Kamenshchik, and U. Moschella, Phys. Rev. D 67, 063509 (2003); A. Dev, D. Jain, and J.E. Alcaniz, ibid. 67, 023515 (2003); J.D. Barrow, Phys. Lett. B 180, 335 (1986); 235, 40 (1990); D. Bazcia and J. Jackiw, Ann. Phys. (N.Y.) 270, 246 (1998); M. Makler, S. Quinet de Oliveira, and I. Waga, Phys. Lett. B 555, 1 (2003); P.F. González-Díaz, ibid. 562, 1 (2003).

[18] D.N. Spergel et al., "First Year Wilkinson Microwave Anisotropy Probe (WMAP) Observations: Determination of Cosmological Parameters," astro-ph/0302209.

[19] J. Yokoyama, Phys. Rev. Lett. 88, 151302 (2002); Int. J. Mod. Phys. D 11, 1603 (2002); R. Schützhold, Phys. Rev. Lett. 89, 081302 (2002).
[20] W. Fischler, A. Kashani-Poor, R. McNees, and S. Paban, J. High Energy Phys. 07, 003 (2001); S. Hellerman, N. Kaloper, and L. Susskind, ibid. 06, 003 (2001); T. Banks, Int. J. Mod. Phys. A 16, 910 (2001); E. Witten, "Quantum gravity in De Sitter space," hep-th/0106109; T. Banks and W. Fischler, "M-theory observables for cosmological space-times," Report No. RUNHETC-2001-, SCIPP-012, UTTG-02-01, hep-th/0102077; Je-An Gu and H-Y.P. Hwang, "The fate of an accelerating universe," astro-ph/0106387.

[21] J.P.S. Lemos, F.S.N. Lobo, and S. Quinet de Oliveira, Phys. Rev. D 68, 064004 (2003)..

[22] R.R. Caldwell, M. Kamionkowski, and N.N. Weinberg, Phys. Rev. Lett. 91, 071301 (2003); G.W. Gibbons, hep-th/0302199; R.R. Caldwell, Phys. Lett. B 545, 23 (2002); S.M. Carroll, M. Hoffman, and M. Trodden, Phys. Rev. D 65, 023509 (2003); V. Sahni and Y. Shtanov, astro-ph/0202346; B. McInnes, J. High Energy Phys. 08, 029 (2002); 12, 053 (2002).

[23] A definition of the exoticity function can be found in Refs. [3] and [21].

[24] M. Bouhmadi and J.A. Jiménez-Madrid (in preparation).

[25] M. Visser, S. Kar, and N. Dadhich, Phys. Rev. Lett. 90, 201102 (2003).

[26] P.F. González-Díaz, Phys. Rev. D 68, 021303(R) (2003). 